\title{
Nutritional supplement use among fitness club participants in Tehran, Iran
}

\begin{abstract}
The aim of this study was to assess nutritional supplement use among fitness club participants in Tehran, Iran. A cross sectional study was conducted in 24 fitness clubs throughout the city of Tehran, Iran. A total of 1625 fitness club participants were recruited to participate in this study. They were asked to complete a self-administered pre-tested questionnaire. Descriptive statistics and chi-square test were performed to determine the characteristics of participants, reasons for supplement use, sources of information and also the influential advisors regarding nutritional supplement use. A high prevalence rate of nutritional supplement use $(66.7 \%)$ was reported. Overall, multivitamin-mineral $(43.8 \%)$ and iron tablets $(30.5 \%)$ were the common nutritional supplements used and only a small number of participants used illegal substances $(0.5 \%)$. Younger participants were more likely to use ergogenic aids, whereas, older participants were more likely to use vitamin D. Males were more likely than females to use creatine and amino acids, whereas, iron tablets and mint water were more common among females. Also, males were more likely to use nutritional supplements for increasing energy, whereas, females were more likely to use nutritional supplements for nutritional deficiencies. In conclusion, a high prevalence rate of nutritional supplement use was seen among participants.
\end{abstract}

Keyword: Nutritional supplements; Fitness clubs; Adults 